\title{
Three-dimensional Kaon Source Imaging from STAR Experiment at RHIC
}

\author{
Michal Šumbera* for the STAR Collboration \\ Nuclear Physics Institute ASCR, 25068 Řež, Czech Republic \\ E-mail: sumbera@ujf.cas.cz
}

Three-dimensional (3-D) source imaging techniques in conjunction with detailed model comparisons have shown the viability of disentangling the spatio-temporal information contained in two-pion interferometric measurements from ultra-relativistic heavy ion collisions. This has led to the observation of non-Gaussian tails in the 3-D pion source function and the extraction of finite pion emission duration at RHIC energies [1]. The STAR Collaboration has recently also extracted the 3-D kaon source function from a high statistics two-kaon interferometric measurement from $\mathrm{Au}+\mathrm{Au}$ collisions at $\sqrt{s_{N N}}=200 \mathrm{GeV}$. Such measurement offers a window into the fireball freeze-out dynamics with a much cleaner probe with smaller resonance decay contributions than for the pion case. The extracted kaon source function is found to have an essentially ellipsoidal shape. The source function can be adequately reproduced by the Therminator event generator[2] with a fireball lifetime $\tau_{0}=8 \mathrm{fm} / \mathrm{c}$ and instantaneous kaon emission. Kaon emission is deemed to occur over a different time-scale and from a different freeze-out hypersurface than pion emission, implying a changing dynamics during the evolution of the fireball.

The 2011 Europhysics Conference on High Energy Physics, EPS-HEP 2011,

July 21-27, 2011

Grenoble, Rhône-Alpes, France

\footnotetext{
* Speaker.
} 
We report on a full 3-D extraction of the kaon source function from a high statistics dataset of $20 \%$ most central Au+Au collisions at $\sqrt{s_{N N}}=200 \mathrm{GeV}$. About 4.6 million and 16 million events collected by the STAR experiment at RHIC during the years 2004 and 2007, respectively, were used for this analysis. Charged tracks were detected in the STAR Time Projection Chamber (TPC) [3], covering \pm 1.8 units of pseudo-rapidity, surrounded by a solenoidal magnet providing a nearly uniform magnetic field of 0.5 Tesla along the beam direction. The $\mathrm{d} E / \mathrm{d} x$ information from the TPC was used to select charged kaons with rapidity $|y|<0.5$ and transverse momenta $0.2<p_{T}<0.4$ $\mathrm{GeV} / c$. Presented results are based on analysis of 117 million like-charge kaon pairs.

The 3-D correlation function $C(\mathbf{q})=N_{\mathrm{fgd}}(\mathbf{q}) / N_{\mathrm{bkg}}(\mathbf{q})$ was constructed as a ratio of 3-D relative momentum distribution for $K^{+} K^{+}$and $K^{-} K^{-}$pairs in the same event $N_{\text {fgd }}(\mathbf{q})$ to that from mixed events $N_{\mathrm{bkg}}(\mathbf{q})$. Here, $\mathbf{q}=\frac{1}{2}\left(\mathbf{p}_{\mathbf{1}}-\mathbf{p}_{\mathbf{2}}\right)$, where $\mathbf{p}_{\mathbf{1}}$ and $\mathbf{p}_{\mathbf{2}}$ are the momentum 3-vectors in the pair center of mass system (PCMS). A right-handed Cartesian coordinate system with $z$ (long) parallel to the beam direction, $x$ (out) pointing in the direction of the pair total transverse momentum $\mathbf{k}_{\mathbf{T}}$ and $y$ (side) perpendicular to $x$ and $z$ is used. $C(\mathbf{q})$ is flat and normalized to unity over $60<|\mathbf{q}|<$ $100 \mathrm{MeV} / c$.

The technique [4] exploited to extract the 3-D kaon source function was previously applied in the first 3-D extraction of the pion source function [1]. It involves the decomposition of 3-D kaon correlation function $C(\mathbf{q})$ into a basis of Cartesian surface-spherical harmonics $A_{\alpha_{1} \ldots \alpha_{l}}^{l}\left(\Omega_{\mathbf{q}}\right)$

to yield the correlation moments

$$
C(\mathbf{q})-1 \equiv R(\mathbf{q})=\sum_{l, \alpha_{1} \ldots \alpha_{l}} R_{\alpha_{1} \ldots \alpha_{l}}^{l}(q) A_{\alpha_{1} \ldots \alpha_{l}}^{l}\left(\Omega_{\mathbf{q}}\right)
$$

$$
R_{\alpha_{1} \ldots \alpha_{l}}^{l}(q)=\frac{(2 l+1) ! !}{l !} \int \frac{d \Omega_{\mathbf{q}}}{4 \pi} A_{\alpha_{1} \ldots \alpha_{l}}^{l}\left(\Omega_{\mathbf{q}}\right) R(\mathbf{q})
$$

Eq. (1) was truncated at $l=4$ and expressed in terms of independent moments only. As expected from symmetry considerations, odd moments were consistent with zero within statistical uncertainty; higher order moments were negligible. Up to order 4, there are 6 independent moments: $R^{0}, R_{x 2}^{2}, R_{y 2}^{2}, R_{x 4}^{4}, R_{y 4}^{4}$ and $R_{x 2 y 2}^{4}\left(R_{x 2}^{2}\right.$ is shorthand for $\left.R_{x x}^{2}\right)$. They were extracted as a function of $q$, by fitting the truncated series to the measured $C(\mathbf{q})$ with the moments as the parameters of the fit. The statistical error on the moments reflect the statistical error on $C(\mathbf{q})$. Other moments were obtained from independent ones [4].

The correlation moments $R_{\alpha_{1} \ldots \alpha_{l}}^{l}$ up to order $l=4$ for mid-rapidity $(|y|<0.5)$, low transverse momentum $\left(0.2<k_{T}<0.36 \mathrm{GeV} / c\right)$ kaon pairs are shown on the left panel of Figure 1 (open circles). In panel (a), $R^{0}(q)$ is compared to the 1D correlation function $R(q)=C(q)-1$ (solid circles); both represent angle-averaged correlation functions, but $R^{0}(q)$ is obtained via Eq. (2) while $R(q)$ is evaluated directly from the one-dimensional correlation function. Their mutual agreement underlines the absence of any significant angular acceptance issues and attests to the reliability of the moment extraction technique. Panels (b)-(f) shows that contributions decrease with increasing $l$ in each direction and are relatively small for $l=4$. This justifies truncating Eq. (1) at $l=4$.

The probability $S(\mathbf{r})$ to emit a pair of particles with a pair separation vector $\mathbf{r}$ in the PCMS is related to $C(\mathbf{q})[6,7]$ :

$$
C(\mathbf{q})-1 \equiv R(\mathbf{q})=\int d \mathbf{r} K(\mathbf{q}, \mathbf{r}) S(\mathbf{r})
$$

The kernel $K(\mathbf{q}, \mathbf{r})$ incorporates Coulomb interaction and Bose-Einstein symmetrization (strong interaction is assumed to be negligible for identical charged kaons). The 3-D source function $S(\mathbf{r})$ 
can be extracted by directly fitting $C(\mathbf{q})$ with a trial functional form for $S(\mathbf{r})$. Since $C(\mathbf{q})$ has been decomposed into its independent moments, this corresponds to a simultaneous fit of the six independent moments with the trial functional form. A 4-parameter ellipsoid (three-dimensional Gaussian) fit gives a reasonable result $\left(\chi^{2} / \mathrm{ndf}=1.7\right)$. The solid curve in left panel of Fig. 1 shows the 4 parameters $\left(\lambda, R_{x}, R_{y}, R_{z}\right)$ fit to the independent moments with an empirical ellipsoid function

$$
S^{G}\left(r_{x}, r_{y}, r_{z}\right)=\frac{\lambda}{(2 \sqrt{\pi})^{3} R_{x} R_{y} R_{z}} \exp \left[-\left(\frac{r_{x}^{2}}{4 R_{x}^{2}}+\frac{r_{y}^{2}}{4 R_{y}^{2}}+\frac{r_{z}^{2}}{4 R_{z}^{2}}\right)\right] .
$$

The middle panel of Fig. 1 displays the kaon correlation function profiles (stars) in the $x, y$ and $z$ directions $\left(C\left(q_{x}\right) \equiv C\left(q_{x}, 0,0\right), C\left(q_{y}\right) \equiv C\left(0, q_{y}, 0\right)\right.$ and $\left.C\left(q_{z}\right) \equiv C\left(0,0, q_{z}\right)\right)$, respectively, obtained by summation of the relevant correlation moments up to order $l=4$. Note the expected BoseEinstein enhancement peak at $q \approx 20 \mathrm{MeV} / \mathrm{c}$ and the dip as $q \rightarrow 0$ due to the Coulomb repulsion. The correlation profiles from the data are well represented by the corresponding profiles from the ellipsoidal fit (blue line) indicating that the trial ellipsoidal shape for the kaon source function captures the essential components of the actual source function.
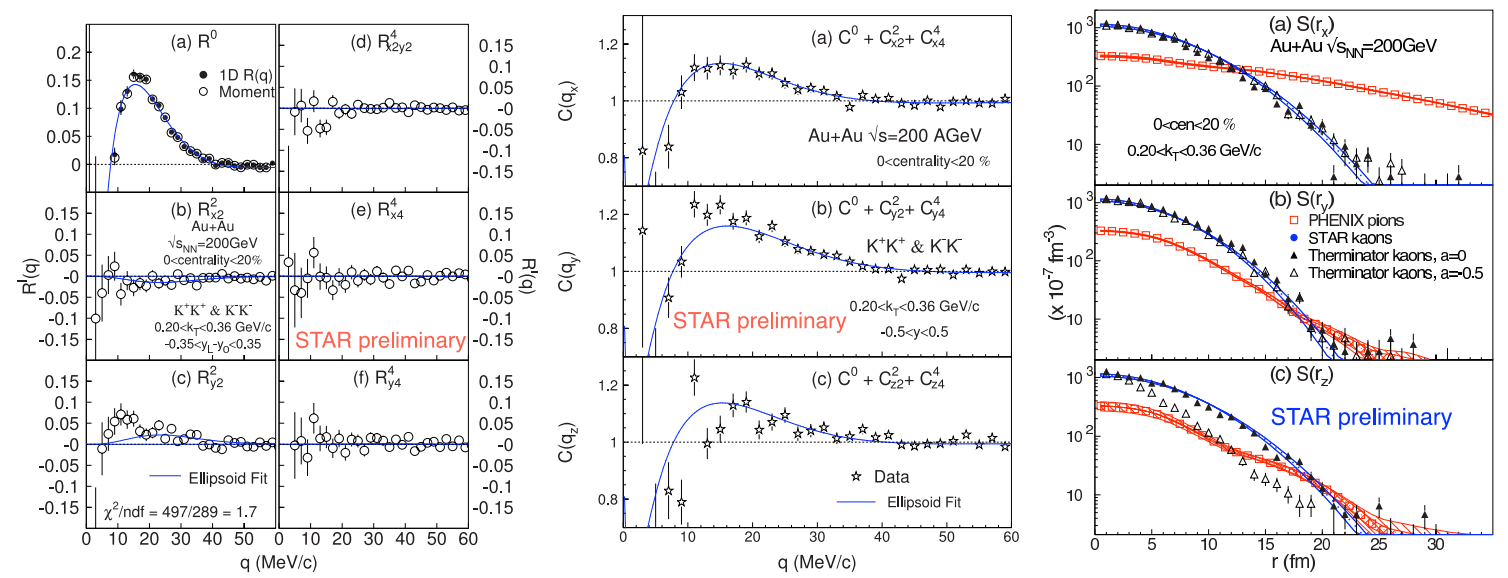

Figure 1: Left: Experimental correlation moments $R^{l}(q)$ for $l=0$, 2, 4. Panel (a) also shows a comparison between $R^{0}(q)$ and $R(q)$ (open and full circles). Systematic errors are smaller than the statistical errors. The solid curve represents the ellipsoid fit. Middle: kaon correlation function profiles (stars) (a) $C\left(q_{x}\right) \equiv$ $C\left(q_{x}, 0,0\right),(b) C\left(q_{y}\right) \equiv C\left(0, q_{y}, 0\right)$ and (c) $C\left(q_{z}\right) \equiv C\left(0,0, q_{z}\right)$ in the $x, y$ and $z$ directions. The curve denotes the ellipsoidal fit profiles. Right: Comparison between 3-D pion source function profiles [1] (squares) and 3-D kaon source function (circles) together with the Therminator predictions for the kaons.

The right panel shows the extracted source function profiles in the $x, y$ and $z$ directions $\left(S\left(r_{x}\right) \equiv\right.$ $S\left(r_{x}, 0,0\right), S\left(r_{y}\right) \equiv S\left(0, r_{y}, 0\right)$ and $\left.S\left(r_{z}\right) \equiv S\left(0,0, r_{z}\right)\right)$ obtained via the ellipsoid fit to the correlation moments (blue dots). The two blue solid curves represent the statistical error band arising from the statistical errors on the 3-D Gaussian fit parameters. The Gaussian shape of kaon source function profiles contrast sharply with data on pions [1] shown as squares on the same figure. The kaon source function profiles are generally narrower in width and more intense for $r \lesssim 15 \mathrm{fm}$ than those for pions. This is a consequence of the higher abundance of pions coming from resonance decays compared to kaons where the resonance contribution is much less. Hence, the pion source function profiles are more extended and therefore less intense than those for kaons.

On the same panel the kaon data are compared to the Monte Carlo event generator Therminator [2] (triangles). Basic ingredients of the model are: (1) Longitudinal boost invariance; (2) Blast- 
wave expansion in the transverse direction with velocity profile semilinear in transverse radius $\rho$ [8]: $v_{r}(\rho)=\left(\rho / \rho_{\max }\right) /\left(\rho / \rho_{\max }+v_{t}\right)$, where the value $v_{t}=0.445$ of the dimensionless parameter $v_{t}$ is used in order to reproduce the measured average transverse velocity [9]; (3) After a proper lifetime $\tau$, a thermal emission of particles occurs from the source elements distributed in a cylinder of infinite longitudinal size and finite transverse dimension $\rho_{\max }$. At the point of source breakup, all particle emissions occur from a freeze-out hypersurface defined in $\rho$ - $\tau$ plane as $\tau=\tau_{0}+a \rho$. Particles emitted from a generic source element with coordinates $(z, \rho)$ have emission time in the laboratory frame $t=\sqrt{\left(\tau_{0}+a \rho\right)^{2}+z^{2}}$. Since each source element is defined by only one value of the proper breakup time $\tau$, all particle emissions from this source element happen instantaneously in the rest frame of the source element and the proper emission duration is 0 .

Using a set of thermodynamic parameters previously tuned to fit charged pion and kaon spectra [10], mid-rapidity kaon pairs at low $k_{T}$ were obtained from Therminator with all known resonance decay processes on and off. They were then boosted to the PCMS to obtain source function profiles for comparison with corresponding profiles from the data.

While the extracted characteristics of the expanding fireball are consistent with those obtained from two-pion interferometry [1], the dynamics behind the emission of the two probes cannot be more different: pions are emitted with a finite emission duration from a freeze-out hypersurface bearing a negative $\rho-\tau$ correlation coefficient $a(-0.5)$ [1] whereas kaons freeze-out instantaneously in the source element rest frame from a hypersurface devoid of any space-time correlation $(a=0)$. This fact is borne out by Therminator calculations with $a=-0.5$ shown on the right panel: the model with a negative $\rho-\tau$ correlation (triangles) simply cannot reproduce the kaon source function profiles in all three directions simultaneously. Hence, pions are emitted from the outer surface of the fireball inwards with finite emission duration while kaons freeze out instantaneously from all parts of the fireball. Such a situation can result from the changing dynamics associated with the evolution of the expanding fireball [11]: since kaons and pions are emitted over different time intervals, they could be subjected to different dynamics at various stages of the expansion.

\section{References}

[1] S. Afanasiev et al. (PHENIX Collaboration) Phys. Rev. Lett. 100, 232301 (2008).

[2] A. Kisiel et al., Comput.Phys.Commun. 174, 669 (2006).

[3] K. H. Ackermann et al. (STAR Collaboration) Nucl. Instrum. Meth. A 499, 624 (2003).

[4] P. Danielewicz and S. Pratt, Phys.Lett. B 618, 60 (2005); Phys.Rev. C 75034907 (2007).

[5] S. Afanasiev et al. (PHENIX Collaboration) Phys. Rev. Lett. 103, 142301 (2009).

[6] M. Gyulassy, S. K. Kauffmann, L. W. Wilson, Phys.Rev. C 20, 2267 (1979).

[7] R. Lednicky, V. L. Lyuboshitz, Sov.J.Nucl.Phys. 35, 770 (1982).

[8] A. Kisiel, Brazilian Journal of Physics, 37, 3A, p.917 (2007).

[9] B. I. Abelev et al. (STAR Collaboration),Phys. Rev. C 79, 034909 (2009).

[10] A. Kisiel et al., Phys. Rev. C 73, 064902 (2006).

[11] Yu. A. Karpenko and Yu. M. Sinyukov, Phys.Rev. C 81, 054903 (2010). 